\title{
Mycoplasma pneumoniae induced popliteal artery thrombosis treated with urokinase
}

\author{
C U Joo, J S Kim, Y M Han
}

\begin{abstract}
A 5 year old boy with serological and clinical evidence of Mycoplasma pneumoniae infection, which was complicated by popliteal artery thrombosis, is described. Intra-arterial urokinase, in conjunction with medical treatment, resulted in clinical recovery and angiographic resolution of the thrombus. The variety of extrapulmonary complications associated with the $M$ pneumoniae infections continues to broaden. Thrombolytic therapies should be considered when similar clinical circumstances arise.
\end{abstract}

(Postgrad Med f 2001;77:723-724)

Keywords: Mycoplasma pneumoniae, arterial thrombosis; urokinase

Mycoplasma pneumoniae is a common cause of respiratory disease in children and adults. The incidence of infection peaks among individuals 10 years of age, with the disease being less common in those under 5 years and over 40 years of age. In general, mycoplasma disease is mild and self limited, although infections may occasionally be associated with a variety of extrapulmonary conditions, although severe complications are rare. Popliteal artery thrombosis is an extremely rare complication of the infection. We report a case of popliteal artery thrombosis in a child with clinical and serological evidence of $M$ pneumoniae infection and the clinical course of recovery after intra-arterial infusion of urokinase.

\section{Case report}

A previously well 5 year old Korean boy was admitted to a local hospital with a 10 day history of intermittent fever, anorexia, and non-productive cough with a presumptive diagnosis of $M$ pneumoniae pneumonia. He was transferred to the Chonbuk National University Hospital because of acute onset of right leg pain accompanied by pallor and coldness. He had been on azithromycin $(10 \mathrm{mg} / \mathrm{kg} /$ day for three days). An examination at the time of the transfer revealed a febrile child with a diminished breath sound over the right upper chest and a cool and pale right leg. The popliteal and pedal pulses in the right leg were not palpable. Neurological examination was normal. On admission, a chest $x$ ray film showed an ill defined consolidation in the right upper lobe, compatible with $M$ pneumoniae pneumonia. The haemoglobin concentration was $113 \mathrm{~g} / \mathrm{l}$ and white blood cell count was $14.8 \times 10^{9} / 1$. The differential count was neutrophils $72 \%$ and lymphocytes $17 \%$. The platelet count was $480 \times 10^{9} / 1$
Because of the perceived urgency of the situation, an emergency angiogram was obtained. The catheter was introduced through the left femoral artery and advanced into the aortic bifurcation where an injection of the contrast media (Visipaque $15 \mathrm{ml}$; Nycomed Ireland Ltd, Cork, Ireland) was made. The angiogram showed non-opacification of the right popliteal artery and its distal branches (fig 1). The catheter was placed in the aortic bifurcation and a 100000 units of urokinase infusion was done for one hour. A repeat angiogram at this time showed a marked improvement in the filling of the distal arteries. Thus, the catheter and sheath were removed and haemostasis accomplished. Urokinase was administered systemically at a dose of 70000 units/hour for the next 12 hours. After the infusion of urokinase, the coldness of the leg gradually improved, and the dorsalis pedis pulse became palpable.

At the time of discharge on the 12th hospital day, all the signs of arterial occlusion were resolved. Follow up magnetic resonance angiogram showed normal visualisation of the both popliteal arteries, and the anterior tibial, posterior tibial, and peroneal arteries (fig 2). We checked protein $\mathrm{C}$, protein $\mathrm{S}$, and other coagulation factors to study the cause of thrombosis. The coagulation profile revealed the following:

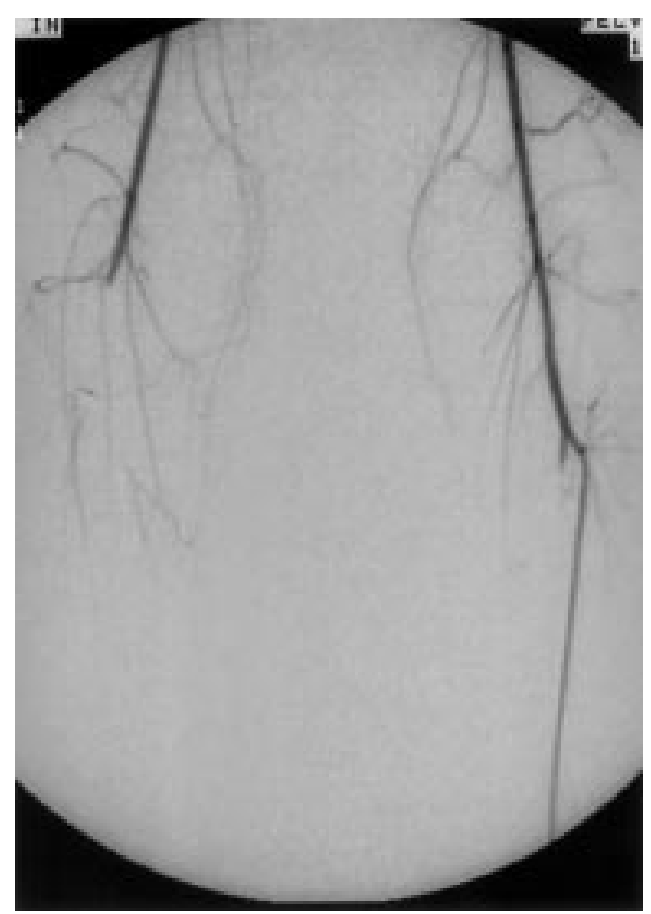

Figure 1 Lower extremity angiogram showing abrupt cutting of the right popliteal artery and non-visualisation of the anterior tibial, posterior tibial and peroneal arteries, which are due to thrombi. There are no-filling contrasts in left posterior tibial and peroneal arteries. 


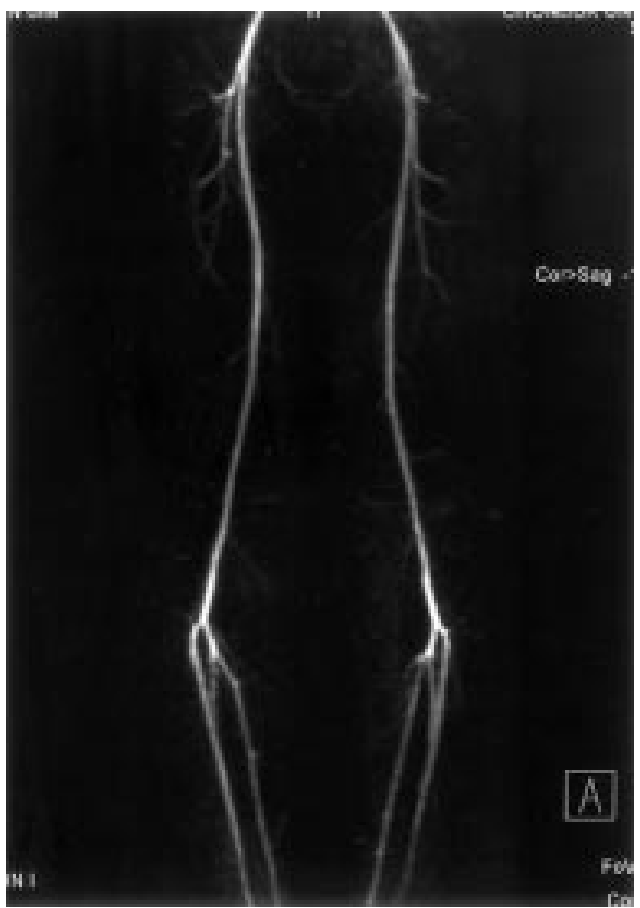

Figure 2 Follow up magnetic resonance angiogram showing normal visualisation of the both lower extremity artery.

free protein S 63\% (normal range 60\%-90\%), total protein S $79 \%(70 \%-140 \%)$, protein C $81 \%(73 \%-142 \%)$, antithrombin III 22.6 $\mathrm{mg} / \mathrm{dl}$ (20-28), fibrinogen $2.9 \mathrm{~g} / 1$ (2.0-4.0), and prothrombin/activated partial thromboplastin time normal. Acute $M$ pneumoniae infection was confirmed with a positive $\operatorname{IgM}$ and a subsequent rise in the antibody titre from $1: 32$ to $1: 256$.

\section{Discussion}

This is a case of popliteal artery thrombosis associated with $M$ pneumoniae infection, which was successfully treated with intra-arterial urokinase infusion. To the best of our knowledge, this is the first report in English literature: there has been one similar report in the French literature. ${ }^{1}$

$M$ pneumoniae is known to cause a mild disease of the upper respiratory tract and pneumonia, with a peak incidence between 5 and 25 years of age. Although serious pulmonary and extrapulmonary complications are known to occur rarely, there have been an increasing number of reports of such complications. The extrapulmonary manifestations of the $M$ pneumoniae infection are diverse and involve nearly every organ system. Complications reported to date include neurological, haematological, renal, dermatological, perimyocardial, gastrointestinal, and musculoskeletal dysfunction. The complications described have occurred one to 23 days after the onset of respiratory tract symptoms. ${ }^{2}$
Summary/learning points

- M pneumoniae is known to cause respiratory infections including pneumonia and extrapulmonary complications.

- The exact mechanisms of extrapulmonary manifestations of the infection are obscure. The development of autoantibodies and immune complex during the course of the infection has been suggested. Lipoglycans formed by the organisms may be responsible for intravascular thrombosis.

- Popliteal artery thrombosis is an extremely rare extrapulmonary manifestation of the $M$ pneumoniae infection.

- Early intra-arterial urokinase infusion is effective in the treatment of the thrombosis.

Despite the numerous reports detailing the extrapulmonary manifestations associated with $M$ pneumoniae infection, the exact mechanism by which the extrapulmonary injury occurs has not yet been established. Possible mechanisms include direct invasion by the organism, toxin production, autoantibody and immune complex formation, microthrombosis, and impaired immunity. ${ }^{2}$

Although intravascular coagulation disorders have been reported during the mycoplasma infection in a few cases, the pathogenic mechanisms are obscure. In in vitro experimental studies, Fumarola suggested that lipoglycans from some mycoplasma species, including $M$ pneumoniae, could induce procoagulant activity (tissue factor-like ability) by human mononuclear cells. ${ }^{3}$ The potent triggering coagulation activity of the lipoglycans may explain the mechanism of the intravascular coagulation associated the mycoplasma infection. Other factors including antithrombin III deficiency (congenital or acquired), deficiency of protein $\mathrm{C}$ or $\mathrm{S}$, or both, should be investigated to identify possible contributors to the pathogenic mechanisms of the thrombosis associated with the disease. ${ }^{45}$

We thank Dr Myung K Park, Department of Pediatrics, the University of Texas Health Science Center at San Antonio, Texas, for his assistance with this manuscript.

1 Ducloux G, Defaux D, Folliot JP, et al. [Femoral artery thrombosis associated with Mycoplasma pneumoniae infection]. LARC Med 1981;1:42-4.

2 Moskal MJ. Mycoplasmal infections. Pulmonary and extrapulmonary manifestations. Postgrad Med 1987;82:104-12.

3 Fumarola D. Intravascular coagulation and Mycoplasma pneumoniae infection. Pediatr Infect Dis 1997;16:1012-3. 4 Creagh MD, Roberts IF, Clark DJ, et al. Familial antithrombin III deficiency and Mycoplasma pneumoniae pneumonia. 7 Clin Pathol 1991;44:870-1.

5 Peyton BD, Cutler BS, Stewart FM. Spontaneous tibial artery thrombosis associated with varicella pneumonia and free protein S deficiency. $\mathcal{F}$ Vasc Surg 1998;27:563-7. 